\title{
Expression analysis and regulation of general transcription repressor, TaDr1, in bread wheat under drought
}

\author{
Zotova L. ${ }^{*}$, Jatayev S. ${ }^{1}$, Kurishbayev A. ${ }^{1}$, Langridge P. ${ }^{2,}$, Schramm C. ${ }^{4}$, Jenkins C. ${ }^{4}$, \\ Soole K. ${ }^{4}$, Shavrukov Y. ${ }^{4}$ \\ ${ }^{1}$ Faculty of Agronomy, S. Seifullin Kazakh AgroTechnical University, Nur-Sultan, Kazakhstan \\ ${ }^{2}$ Wheat Initiative, Julius Kühn-Institute, Berlin, Germany \\ ${ }^{3}$ University of Adelaide, SA, Australia \\ ${ }^{4}$ College of Science and Engineering, Biological Sciences, Flinders University, SA, Australia \\ *e-mail:lupezo_83@mail.ru
}

Introduction and Aim: The key factor in achieving increased yields of spring wheat is the enrichment of the gene-pool using germplasm collections from foreign countries. The study and introgression of wheat germplasm accessions from various ecological and geographical origins is important for the wheat breeding process. These genetic materials can be used to study candidate genes responsive to abiotic stress and associated with agronomically important traits. The study of genetic polymorphism and expression of the transcriptional repressor gene, TaDrl, together with the TaVrn1 and TaFT1 genes which control the transition to the reproductive stage and flowering, can help to better understand the mechanism of drought tolerance and improve wheat yields in Northern Kazakhstan.

Results: High- and low-yielding wheat accessions were identified through screening of the world spring wheat germplasm collection via environmental tests in the local environment of Northern Kazakhstan with its harsh continental climate. As a result of molecular studies, the Amplifluor-like SNP marker KATU-W62 was developed based on TaDrl gene polymorphism, for accurate wheat genotyping. Increased expression of TaDrl was observed in response to drought, while the level of expression was higher in high-yielding wheat samples than in low-yielding accessions. Additionally, it was revealed that TaDrl is associated with two genes controlling wheat plant development, TaVrn1 and TaFT1. All three studied genes, TaDr1, TaVrn1, and TaFT1, had similar changes in expression in response to drought, which is of particular interest for further research.

Acknowledgments: This study was supported by the Ministry of Education and Science, Kazakhstan, Research program BR05236500 (SJ). 